\title{
Does Vitamin C and E Supplementation Impair the Favorable Adaptations of Regular Exercise?
}

\author{
Michalis G. Nikolaidis, ${ }^{1}$ Chad M. Kerksick, ${ }^{2}$ Manfred Lamprecht, ${ }^{3}$ and Steven R. McAnulty ${ }^{4}$ \\ ${ }^{1}$ Department of Physical Education and Sports Science at Serres, Aristotle University of Thessaloniki, 62110 Serres, Greece \\ ${ }^{2}$ Department of Health, Exercise and Sport Sciences, University of New Mexico, Albuquerque, NM 87109, USA \\ ${ }^{3}$ Centre for Physiological Medicine, Medical University of Graz, Harrachgasse 21/II, 8010 Graz, Austria \\ ${ }^{4}$ Department of Health, Leisure, and Exercise Science, Appalachian State University, Boone, NC 28608, USA \\ Correspondence should be addressed to Michalis G. Nikolaidis, nikolaidis@auth.gr
}

Received 1 April 2012; Revised 18 June 2012; Accepted 20 June 2012

Academic Editor: Felipe Dal-Pizzol

Copyright () 2012 Michalis G. Nikolaidis et al. This is an open access article distributed under the Creative Commons Attribution License, which permits unrestricted use, distribution, and reproduction in any medium, provided the original work is properly cited.

\begin{abstract}
The detrimental outcomes associated with unregulated and excessive production of free radicals remains a physiological concern that has implications to health, medicine and performance. Available evidence suggests that physiological adaptations to exercise training can enhance the body's ability to quench free radicals and circumstantial evidence exists to suggest that key vitamins and nutrients may provide additional support to mitigate the untoward effects associated with increased free radical production. However, controversy has risen regarding the potential outcomes associated with vitamins $\mathrm{C}$ and $\mathrm{E}$, two popular antioxidant nutrients. Recent evidence has been put forth suggesting that exogenous administration of these antioxidants may be harmful to performance making interpretations regarding the efficacy of antioxidants challenging. The available studies that employed both animal and human models provided conflicting outcomes regarding the efficacy of vitamin $\mathrm{C}$ and $\mathrm{E}$ supplementation, at least partly due to methodological differences in assessing oxidative stress and training adaptations. Based on the contradictory evidence regarding the effects of higher intakes of vitamin $\mathrm{C}$ and/or $\mathrm{E}$ on exercise performance and redox homeostasis, a permanent intake of non-physiological dosages of vitamin $\mathrm{C}$ and/or E cannot be recommended to healthy, exercising individuals.
\end{abstract}

\section{Introduction}

The antioxidant vitamins $\mathrm{C}$ (ascorbic acid) and $\mathrm{E}(\alpha, \beta$, $\delta$, and $\gamma$ tocopherols and tocotrienols) are involved in protecting cellular organelles from oxidative damage [1, $2]$. Exercise can increase free radical production by 2 to 4 -fold [3] and produce changes in redox status which may exert oxidative stress on muscles and other tissues leading to alteration of lipids, proteins, and genetic material [4]. Short-term exercise results in temporary increases in concentrations of oxidized products [5], but habitual exercise may result in an augmented endogenous antioxidant system and a reduction in oxidized products $[4$, $6,7]$. Supporting endogenous antioxidant defense systems with additional oral doses of antioxidants has received much attention as a strategy to reduce oxidative stress, decrease muscle damage, and improve exercise performance.

Indeed, a significant number of athletes, including elite athletes, consume vitamin supplements seeking beneficial effects on performance [8]. However, recently, there is a growing evidence of the negative effects of antioxidant supplementation on exercise performance in both animal and human studies $[9,10]$. In light of the results of these prolific studies $[9,10]$, it was concluded that vitamin C and/or $\mathrm{E}$ interfere with the adaptive responses to endurance exercise training. More studies followed trying to delineate the possible effects of antioxidant supplementation on adaptations in exercise performance and/or redox homeostasis [11-17]. In this context, we performed the present literature analysis to evaluate whether antioxidant vitamin $\mathrm{C}$ and/or $\mathrm{E}$ supplementation affect the favorable adaptations of exercise. 


\section{Criteria for Study Inclusion and Methodological Issues}

There are too many studies to properly analyze the effect of antioxidants other than vitamins $\mathrm{C}$ and $\mathrm{E}$ (e.g., [18-25]). We have chosen to focus only on vitamins $\mathrm{C}$ and $\mathrm{E}$ for three reasons. First, because vitamins $\mathrm{C}$ and $\mathrm{E}$ are by far the two most well-characterized antioxidants. Second, because both vitamins $\mathrm{C}$ and $\mathrm{E}$ exert their antioxidant action via a common mechanism, that is by donating a hydrogen atom to a free radical [26]. Third, because there is a well-described dependency between these two antioxidants, since vitamin C recycles vitamin E via the tocopheroxyl radical [27]. Finally, it is important to consider that the number of antioxidants, aside from vitamins $\mathrm{C}$ and $\mathrm{E}$ is huge and include many illcharacterized molecules with debatable antioxidant function [28].

Eleven studies, consisting of both rat and human work, were selected for analysis (Tables 1 and 2). No restrictions were made regarding the type of exercise used (e.g., endurance or resistance). In addition, a relevant study that employed a nonphysiological exercise model, namely, in situ muscle stimulation [13], is also included, mainly because it provides unlimited access to skeletal muscle and offers mechanistic insights for the data derived from physiological models.

In order for a study to be included in the present analysis the following criteria should have been met:

(1) some manner of simultaneous implementation of chronic exercise and vitamin $\mathrm{C}$ and/or vitamin $\mathrm{E}$ supplementation for more than three weeks. This time period was judged sufficient to allow exercise training adaptations to appear;

(2) recruitment of untrained or moderately trained individuals to allow for adequate physiological training adaptations;

(3) use of appropriate control group(s);

(4) no use of other antioxidant/nutrient supplementation (e.g., carotene, lipoic acid) except for vitamin C, vitamin $\mathrm{E}$, or a combination of the two;

(5) measurement of unambiguous biochemical (e.g., activity of mitochondrial enzymes or insulin sensitivity) and/or physiological endpoints of chronic exercise (e.g., $\mathrm{VO}_{2}$ max or muscle strength).

\section{Effect of Vitamin C and/or E Supplementation on Adaptations to Chronic Exercise}

Asha Devi et al. $[29,30]$ examined the effect of vitamin $\mathrm{E}$ ( $\alpha$-tocopherol; $50 \mathrm{IU} / \mathrm{kg}$ bw/day) supplementation on the adaptations induced by 12 weeks of endurance swimming in young ( 4 or 8 months), middle-aged (12 months), and old rats ( 22 months). The untrained animals ( 3 males per group) were orally supplemented with vitamin $\mathrm{E}$ and swam for 8.5 weeks. The levels of vitamin $\mathrm{E}$ in the left and right ventricles of the heart of supplemented rats increased 17$44 \%$ at the end of the study. In general, the levels of lipid peroxidation in the heart (determined through thiobarbituric acid reactive substances; TBARS) were lower in the animals who received the daily dosage of vitamin E. Overall, catalase activity increased in the vitamin-E-supplemented hearts except for the very old animals (i.e., 22 months old), where no differences appeared between the supplemented and the nonsupplemented animals. Similarly, superoxide dismutase (SOD) activity in the heart was generally higher in the supplemented animals than the nonsupplemented ones. Swimming training improved the blood lipid profile of rats (i.e., elevated HDL cholesterol and lower LDL cholesterol), and this was generally more evident in the vitamin-Esupplemented rats. Finally, vitamin-E-supplemented rats exhibited higher-endurance capacity from 21 to $31 \%$ compared to the untrained counterparts. The findings of this study suggest that vitamin-E-supplementation may induce an ergogenic effect and promote favorable adaptations to blood lipid profile.

Higashida et al. [11] studied the effect of combined vitamin $\mathrm{C}(750 \mathrm{mg} / \mathrm{kg}$ bw/day) and vitamin $\mathrm{E}(\alpha-$ tocopherol; $150 \mathrm{mg} / \mathrm{kg}$ bw/day) supplementation on the training-induced adaptive responses of muscle mitochondria and insulin sensitivity in untrained rats $(3$ males in the placebo groups aged 3 months and 6 males in the supplemented groups aged 3 months). Two studies were performed whereby a short-term study provided vitamins $\mathrm{C}$ and $\mathrm{E}$ for nine days and the animals swam for the last three days of the vitamin treatment. A longer study provided vitamin supplementation for eight weeks and in conjunction had the animals swim for the last three weeks of supplementation. No measurement of vitamins was performed in plasma or tissues. The last acute exercise session resulted in an $80 \%$ increase in plasma TBARS, a response that was prevented in the rats given vitamin $\mathrm{C}$ and vitamin E. Noteworthy, there was no effect of antioxidant supplementation on the resting levels of lipid peroxidation. Based on this finding, it is probable that the resting levels of many oxidative stress markers (including TBARS) can give much less or no information compared to the ones modified by an acute-exercise session. In other words, it may be easier to find an effect of antioxidant supplementation on redox status after exercise than at rest. This may render exercise as a convenient model to study redox status homeostasis. In general, both the short and the long exercise regimes induced similar adaptations. Briefly, swimming increased the protein levels of copper zinc SOD (CuZnSOD), manganese SOD (MnSOD) (only after the short-term study), peroxisome proliferator-activated receptor gamma coactivator 1-alpha (PGC-1 $\alpha$ ), and seven mitochondrial proteins (cytochrome oxidase I, cytochrome oxidase IV, citric synthase, ATP synthase, succinate-ubiquinone oxidoreductase, $\mathrm{NADH}$-ubiquinone oxidoreductase, and long-chain acylCoA dehydrogenase). Exercise-induced upregulation of these proteins was unaffected by the vitamin supplementation. In addition, glucose transporter 4 (GLUT4) expression and insulin responsiveness (determined by measuring the glucose analog 2-deoxyglucose in the extracellular space and intramuscularly in an in vitro assay) increased to about the same extent in both control and vitamin-supplemented 


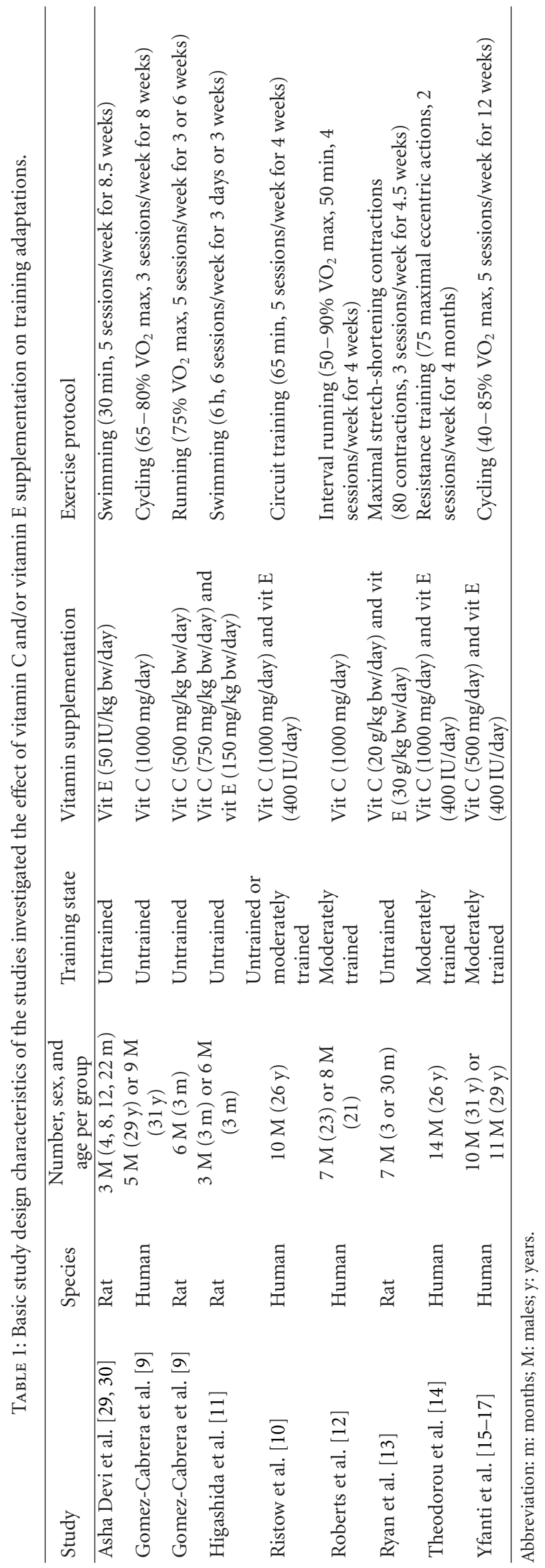




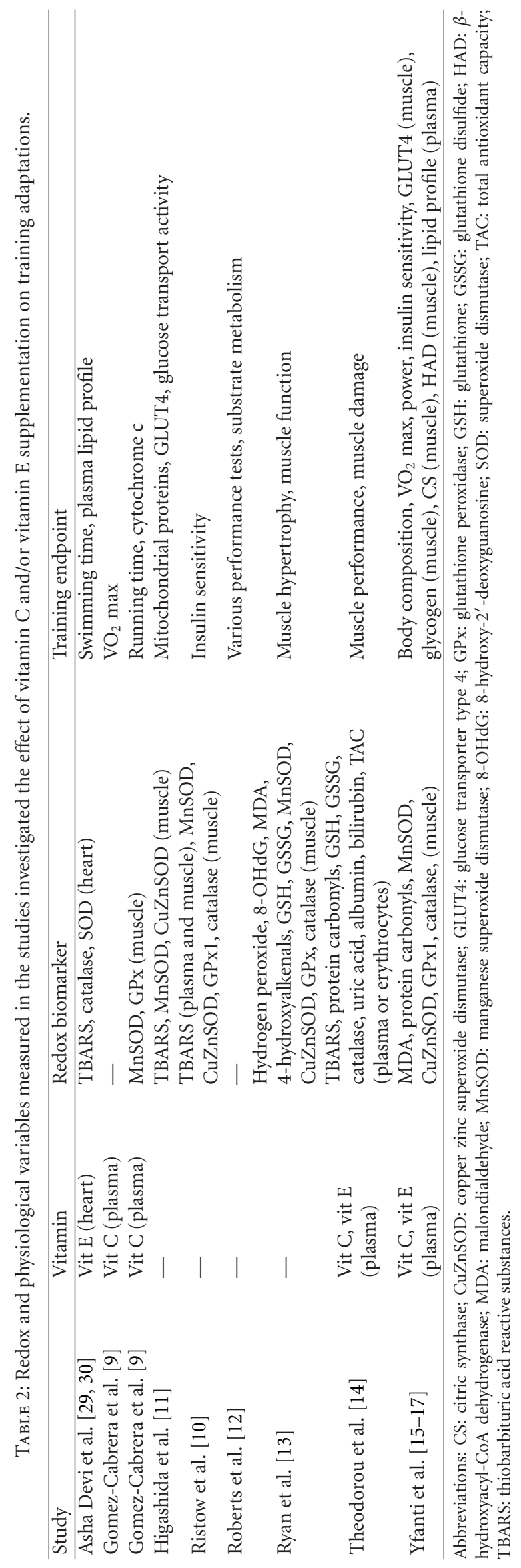


groups. No physiological training endpoint was measured. However, based on the fact that vitamins $\mathrm{C}$ and $\mathrm{E}$ did not affect the adaptive increases in mitochondria as a result of training, it is highly probable that no changes in endurance would have taken place. Based on the findings of this study, vitamin $\mathrm{C}$ and $\mathrm{E}$ supplementation does not have an inhibitory or a promoting effect on the adaptive responses related to mitochondria and glucose metabolism of skeletal muscle to chronic exercise.

Ryan and colleagues [13] explored the effects of chronic electrical stimulation along with vitamin $\mathrm{C}$ and vitamin $\mathrm{E}$ supplementation on redox homeostasis and muscle function in young ( 7 males per group aged 3 months) and old rats ( 7 males per group aged 30 months). The untrained rats were subjected to 80 maximal concentric-eccentric actions per session, three times per week for 4.5 weeks. Vitamin C $(20 \mathrm{~g} / \mathrm{kg}$ bw/day) and vitamin E ( $\alpha$-tocopheryl acetate; $30 \mathrm{~g} / \mathrm{kg}$ bw/day) supplementation began one week before the first electrical stimulation session. The level of vitamins $\mathrm{C}$ and $\mathrm{E}$ in plasma or tissue was not determined. The researchers measured hydrogen peroxide (precursor of hydroxyl radical in the presence of ferrous iron), total and oxidized glutathione (major redox couple), 8-hydroxy-2'deoxyguanosine (8-OHdG; biomarker of oxidized DNA), malondialdehyde (MDA; biomarker of lipid peroxidation) and 4-hydroxyalkenals (biomarker of lipid peroxidation), in muscle. Moreover, they measured the mRNA, protein and activity levels of catalase, CuZnSOD, MnSOD, and glutathione peroxidase $(\mathrm{GPx})$ in muscle. Due to the large number of redox biomarkers determined and the three independent variables tested (i.e., nutrition: supplementation versus no supplementation; exercise: training versus no training; aging: young versus old), it is difficult to reconcile the effects of electrical stimulation and/or vitamin supplementation on redox homeostasis. In general, the levels of hydrogen peroxide, total glutathione, MDA, and 8-OHdG increased in skeletal muscle from young and aged animals after electrical stimulation indicating the presence of oxidative stress. These increases were attenuated or blocked in the vitamin-C- and E-supplemented animals demonstrating the antioxidant effects of vitamins $\mathrm{C}$ and $\mathrm{E}$. In general, the activity of the antioxidant enzymes GPx, catalase, CuZnSOD, and MnSOD either increased or were not affected by electrical stimulation. The effect of vitamin supplementation on the activity of antioxidant enzymes was not uniform. Similarly, the evidence of a disparity among mRNA abundance, protein abundance, and enzyme activity was presented, indicating that it is difficult to predict the antioxidant enzyme activity from mRNA and/or protein data. Electrical stimulation increased the mass of tibialis anterior (the muscle subjected to the stimulation), and this effect appeared in both control and supplemented animals. Vitamin supplementation did not affect force, concentric work, or eccentric work of either resting or stimulated muscles in young rats. In contrast, the positive work of skeletal muscle in aged animals increased only in the vitamin supplemented rats, demonstrating an ergogenic effect of vitamins $C$ and $E$ in this group of animals. In summary, vitamin $\mathrm{C}$ and $\mathrm{E}$ supplementation generally reduced oxidative stress induced by electrical stimulation and improved concentric work in old animals but not in young.

Gomez-Cabrera et al. [9] investigated the effect of vitamin $\mathrm{C}$ supplementation on molecular and physiological adaptations which took place after running training in both animals and humans. In the animal study (6 males per group, 3 months old), untrained rats ran on a treadmill for either three or six weeks while being administered vitamin C orally ( $500 \mathrm{mg} / \mathrm{kg}$ bw/day). Vitamin C supplementation resulted in a threefold increase in plasma vitamin $\mathrm{C}$ concentration when compared to non-vitamin-C-supplemented animals. Vitamin $\mathrm{C}$ supplementation hindered the increase in the mRNA levels of MnSOD and GPx, the protein levels of PGC$1 \alpha$, and the mRNA and protein levels of nuclear respiratory factor 1 (NRF-1) and mitochondrial transcription factor A (mTFA) induced by training. In addition, vitamin C supplementation blunted the increase of cytochrome $\mathrm{c}$ (i.e., a biomarker of mitochondrial biogenesis) in the trained animals. In addition, vitamin $\mathrm{C}$ administration largely hampered the increase in endurance capacity which occurred as a result of training. In fact, the authors reported that control rats increased running endurance by $187 \%$ whereas vitamin-C-supplemented rats increased by just $26 \%$. In the human study ( 5 males in the placebo group aged 29 years and 9 males in the supplemented group aged 31 years), untrained participants cycled for eight weeks while being orally administered vitamin C $(1000 \mathrm{mg} /$ day $)$. Vitamin C supplementation stimulated a 3.8 -fold greater increase in plasma vitamin $\mathrm{C}$ concentration when compared to the non-vitamin-C-supplemented participants. No biomarkers of redox homeostasis or mitochondrial biogenesis were measured in the human study. The effects of the training protocol on $\mathrm{VO}_{2}$ max, were dependent on whether the animals received vitamin $\mathrm{C}$ or not. For example, the nonsupplemented humans experienced a $22 \%$ increase $(P<$ $0.05)$ in $\mathrm{VO}_{2}$ max whereas the participants who received vitamin $\mathrm{C}$ showed a nonsignificant $(P>0.05)$ increase of $11 \%$ in $\mathrm{VO}_{2}$ max. It is worth mentioning that in both the animal and human studies a vitamin-only-supplemented sedentary control group was not included. As a result, it is not possible to discern the isolated effects of vitamin C on the measured variables. Gomez-Cabrera et al. [9] were the only researchers who studied the adaptations to vitamin $\mathrm{C}$ or $\mathrm{E}$ supplementation in two species, rats and humans, concurrently (in fact, this is the only comparative study reviewed in the present article). Comparative exercise studies are indispensable in providing a criterion as to how safe it is to extrapolate findings from animals to humans, which is frequently done by researchers in an effort to compare their findings in animals with those in humans. Gomez-Cabrera et al. [9] found that vitamin C and E supplementation caused similar changes in the adaptations to chronic exercise of both species. To our point of view, though, the major setback in translational redox biology research is the fact that, contrary to the common belief, findings from animal experiments cannot be automatically translated to humans. In fact, to the best of our knowledge, no study has ever investigated whether using laboratory animals is a valid experimental model of human redox biology in vivo. The major conclusion 
of this study is that supplementation with vitamin C markedly lowers the training efficiency in both animals and humans and hinders the training-induced upregulation of antioxidant enzymes in animals.

Ristow et al. [10] evaluated the effects of a combination of vitamin $\mathrm{C}$ and vitamin $\mathrm{E}$ ( $\alpha$-tocopherol) on antioxidant enzymes and insulin sensitivity in the muscle of untrained and moderately trained individuals (10 males per group aged 26 years). Human participants were supplemented with vitamins C (1000 mg/day) and E (400 IU/day) and exercised for 45 minutes in a circuit fashion for four weeks. Skeletal muscle biopsies were obtained before and after the training intervention. Although, the level of vitamins $\mathrm{C}$ and $\mathrm{E}$ in plasma or tissue was not determined, the group that was supplemented prevented the increase in lipid peroxidation (measured through TBARS) in skeletal muscle after a specific short-term exercise regimen lasting three days. Exercise increased the expression of reactive species-sensitive transcriptional regulators of insulin sensitivity (i.e., peroxisomeproliferator-activated receptor gamma $(\operatorname{PPAR} \gamma)$ and PPAR coactivators, PGC- $1 \alpha$ and PGC- $1 \beta$ ) and up-regulated antioxidant enzymes (i.e., CuZnSOD, MnSOD, and GPx1) in both trained and untrained individuals. In addition, exercise training increased insulin sensitivity as measured by glucose infusion rate during a hyperinsulinemic euglycemic clamp. Particularly, regarding the insulin sensitivity, it is of note that the euglycemic-hyperinsulinemic clamps and the muscle biopsies were performed seven days after the end of the training. The improvement in insulin sensitivity in response to training reverses rapidly after cessation of training and is no longer present after seven days [31]. Therefore, the finding of the persistent increase in insulin sensitivity in the control group is hard to explain. It is noteworthy that all of the above effects of exercise training were blocked or reduced by the vitamin $\mathrm{C}$ and $\mathrm{E}$ supplementation. Despite the fact that $\mathrm{VO}_{2}$ max was measured before the initiation of the training, it was not measured at the end of it. Consequently, no conclusion can be drawn whether vitamin $\mathrm{C}$ and $\mathrm{E}$ supplementation also hindered the anticipated improvements in endurance capacity. In addition, a vitamin only sedentary control group was not included. In summary, vitamin $\mathrm{C}$ and $\mathrm{E}$ supplementation prevented the induction of antioxidant enzymes and insulin sensitivity after chronic exercise in both trained and untrained humans.

Roberts et al. [12] determined the effects of four weeks of vitamin C (1000 mg/day) supplementation on the traininginduced improvements in exercise performance of moderately trained humans ( 7 males in the placebo group aged 23 years and 8 males in the supplemented group aged 21 years). A high-intensity interval running protocol lasting four weeks was implemented. The level of vitamin $\mathrm{C}$ before and after the supplementation was not determined. Training improved both $\mathrm{VO}_{2}$ max and performance as measured by several physical fitness tests. In addition and as expected, training increased fat oxidation rate and decreased carbohydrate oxidation rate during acute exercise. Neither of the physical performance tests nor substrate metabolism during exercise were affected by vitamin $\mathrm{C}$ supplementation. In conclusion, vitamin $\mathrm{C}$ supplementation during four weeks of interval training did not affect training-induced improvements in exercise performance of humans.

Theodorou et al. [14] investigated the effects of a combination of vitamin $\mathrm{C}$ and vitamin $\mathrm{E}$ ( $\alpha$-tocopherol) along with an eccentric resistance training on muscle performance and blood redox homeostasis in moderately trained humans (14 males per group aged 26 years). This is the only study that used a resistance exercise protocol instead of an endurance protocol. In fact, an eccentric exercise model was employed to induce redox homeostasis alterations, which is characterized by long (lasting for many days after exercise) and extensive increases in oxidative stress and induce resistance training adaptations [32-34]. This type of exercise was selected for increasing the statistical power to find a significant effect if one existed. In a double-blinded fashion, men received either a daily oral supplement of both vitamin C (1000 mg/day) and vitamin E (400 IU/day) or a placebo for 11 weeks. The subjects performed an eccentricexercise session two times per week for four weeks. Before and after the training protocol, the subjects underwent one session of acute-eccentric exercise to explore the shortterm effects of eccentric exercise in both trained and untrained individuals. The vitamin-supplemented group had 36\% higher plasma vitamin C concentration and 33\% higher plasma vitamin E concentration than the nonvitamin supplemented counterparts. The results failed to support any effect of vitamin supplementation. Acute and chronic eccentric exercise similarly modified muscle damage, muscle performance, blood redox biomarkers, and hemolysis in both the supplemented and the nonsupplemented group. This occurred despite the fact that acute and chronic eccentric exercise induced marked changes in muscle damage, performance, and redox homeostasis.

In a large study published in a series of three articles, Yfanti et al. [15-17] investigated the effect of combined vitamin $\mathrm{C}$ and vitamin $\mathrm{E}$ ( $\alpha$-tocopherol) supplementation along with endurance training on redox homeostasis, training adaptation biomarkers (i.e., muscle glycogen and muscle mitochondrial enzymes), blood lipid profiles, insulin sensitivity, body composition, and endurance performance. Using a double-blinded placebo-controlled design, moderately trained young men received either oral supplementation with vitamin $\mathrm{C}$ and $\mathrm{E}(500 \mathrm{mg} /$ day and $400 \mathrm{IU} /$ day, respectively; 11 males aged 29 years) or placebo (10 males aged 31 years) before and during 12 weeks of bicycle training at a frequency of five days per week. Muscle biopsies along with blood samples were collected before and after training. The vitamin-supplemented group exhibited 37\% higher plasma vitamin $\mathrm{C}$ concentration and $47 \%$ higher plasma vitamin E concentration than the non-vitamin-supplemented group. Lipid peroxidation (assessed through TBARS) in skeletal muscle increased after both acute and chronic exercise irrespective of vitamin supplementation. However, when several postexercise time points were combined by calculating the area under the curve, a significant increase in muscle TBARS appeared only in the vitamin $\mathrm{C}$ and $\mathrm{E}$ group and not in the placebo group. Therefore, it could be inferred that the level of lipid peroxidation in blood plasma was higher overall in the vitamin-supplemented group. These two findings 
indicate prooxidant effects of vitamin supplementation. In general, training had no impact on the mRNA levels or the protein levels of catalase, CuZnSOD, and GPx1. On the other hand, vitamin $\mathrm{C}$ and $\mathrm{E}$ supplementation increased the mRNA levels of CuZnSOD and GPxl. Unexpectedly, vitamin supplementation did not affect the protein levels of these enzymes. This disparity between mRNA and protein abundance agrees with the findings reported by Ryan et al. [13] and indicates the difficulty of predicting antioxidant enzyme responses at the protein level from mRNA data. This study also measured three well-established biochemical markers of endurance training adaptation, namely, glycogen concentration and the activity of citric synthase and $\beta$-hydroxyacyl-CoA dehydrogenase in skeletal muscle. As expected, endurance training increased the content of both glycogen and the mitochondrial enzymes and this effect appeared in both vitamin- and non-vitamin-supplemented rats. Regarding the blood lipid profile, endurance training increased the HDL cholesterol similarly in the two groups, while no effects appeared in the other blood lipids. Insulin-stimulated glucose uptake (using a euglycemichyperinsulinemic clamp) increased similarly in both the vitamin and the placebo group in response to training. In the same way, no differences appeared between the vitamin and the placebo group in the protein content of several insulin cascade molecules. Body composition (i.e., fat mass and fat-free mass determined using dual energy X-ray absorptiometry) were not affected by the training protocol or the vitamin supplementation. Finally, exercise performance (i.e., $\mathrm{VO}_{2}$ max, maximal power output and workload at lactate threshold) increased in both groups independently of vitamin supplementation. In conclusion, the work of Yfanti et al. [15-17] indicates that vitamin $C$ and $E$ supplementation, despite some effects on redox homeostasis, did not affect the biochemical and physiological phenotype of individuals trained strenuously in an endurance fashion for 12 weeks.

\section{Discussion}

The studies included in our paper reveal conflicting results regarding the effects of vitamin $\mathrm{C}$ and $\mathrm{E}$ supplementation on adaptation to chronic exercise. Two publications demonstrated ergolytic (i.e., negative) effects $[9,10]$, six papers yielded no effect [11, 12, 14-17], and two studies reported an ergogenic outcome $[13,29]$. Of the two negative papers, one involved a human component and both provided an animal aspect to the investigation. The rat study was conducted with a very high dose of vitamin C ( $500 \mathrm{mg} / \mathrm{kg}$ body weight/day), which translates to 30-40 grams per day of vitamin C when converted to human body weight [9]. The human studies were conducted with reasonable and otherwise practical dosages: $1000 \mathrm{mg}$ /day of vitamin $\mathrm{C}$ and/or additionally 400 IU of RRR-alpha-tocopherol over three to eight weeks [9, 10]. It is noteworthy that supplementation with just vitamin $\mathrm{C}$ is not comparable to supplementation with a combination of vitamins $\mathrm{C}$ and $\mathrm{E}$, even when concentrations of vitamin $\mathrm{C}$ are similar. In this respect, added vitamin $\mathrm{E}$ might change the impact on redox homeostasis due to its antioxidant and also prooxidant properties [35].
Of the six manuscripts that demonstrated no influence of vitamin $\mathrm{C}$ and $\mathrm{E}$ supplementation on various adaptations, two were rat studies with the remainder being performed with human subjects. Again, dosing issues were prevalent in the rat studies $[9,11,13,29,30]$ whereby dosages of vitamins were approximately $0.5-59$ grams per day of vitamin C and $0.15-88$ grams per day of vitamin E. Although the redox biology between rats and humans is apparently similar and animal studies are often essential before investigating humans, the dosages employed in the rat studies make it difficult to interpret biological relevance for human redox biology.

The human studies showing no effects [14-17] were conducted with reasonable dosages of vitamins C (500$1,000 \mathrm{mg} /$ day) and E (400 IU) over 4-16 weeks. Moreover, Theodorou et al. [14] and Yfanti et al. [15-17] also used the same stereoisomers of alpha-tocopherol, the natural RRR form of vitamin E, and similar surrogate markers of redox homeostasis (protein carbonyls, catalase). In contrast, they tested distinct training regimens and used different outcome measurements to determine their overall outcomes (resistance versus endurance training, muscle performance versus $\mathrm{VO}_{2}$ max, etc.). In comparison to the Ristow paper [10] that showed a negative effect of supplementation, these studies used the same type and dosage of vitamin $\mathrm{E}$ but utilized completely different training regimens.

Two studies revealed ergogenic effects of vitamin E supplementation or a combination of vitamins $\mathrm{E}$ and $\mathrm{C}$ $[13,29]$, both conducted with rats. All of the rat studies under review used high dosages of vitamin $\mathrm{E}$ although Asha Devi et al. $[29,30]$ used the lowest with about 3,500 IU of vitamin E per day over 8.5 weeks when converted to human body weight. The two studies are not comparable to human investigations regarding dosage, training regimen, and outcome measures and therefore, it is difficult to translate these results to humans.

The discrepancy among studies described above regarding the influence of vitamin $\mathrm{C}$ and/or $\mathrm{E}$ supplementation on adaptations in redox homeostasis also applies to the effects of antioxidant supplementation on alterations in redox homeostasis in response to acute exercise. In fact, most of the relevant studies have reported that vitamin $\mathrm{C}$ and/or $\mathrm{E}$ supplementation does not affect redox status [14, 18, 36-40], less studies have reported that attenuates oxidative stress [4145 ] and there are even reports indicating a pro-oxidant effect $[46,47]$. We believe that this "agreement on disagreement" between responses and adaptations to exercise indicates the inherent complexity of redox biochemistry and signifies the difficulty in providing unidirectional predictions after supplementation with antioxidant agents.

Another important methodological issue of the pertinent studies is that all of them employed untrained or recreationally trained individuals (i.e., $\mathrm{VO}_{2} \max 50-58 \mathrm{~mL} / \mathrm{kg} / \mathrm{min}$ [10, 15-17]; Theodorou et al. [14] did not report the physical capacity of the participants). This limitation has important practical applications because most of the individuals taking antioxidant supplements are actually well trained or even professional athletes. This is particularly relevant for the antioxidant supplementation field, since it has been 
repeatedly reported that strenuous bouts of exercise do not result in a significant increase in blood oxidative stress in trained men $[48,49]$. This is probably because participation in a regular exercise program is associated with a chronic upregulation in antioxidant defense [14, 50], which may serve to provide protection against the exercise-induced increase in free radicals. Taking these facts into account, it is possible that the possible negative effects of antioxidant supplementation on performance and redox status of welltrained individuals may be miniscule or absent considering that there is little room for improvement. Besides, none of the pertinent studies have employed females as participants. This is also an important limitation considering that, for example, lower amounts of exercise-induced lipid peroxidation may be produced in females due to increased antioxidant potential as a result of elevated estrogens levels [51].

It is worth mentioning that the properties of specific antioxidants do not necessarily translate to other antioxidants. For example, despite the fact that some studies have reported that vitamin $\mathrm{C}$ [9] or combined vitamin $\mathrm{C}$ and $\mathrm{E}$ supplementation [10] attenuated or prevented the exercise adaptations this does not mean in any way that all types of antioxidants induce negative effects. Indeed, the disparity noted among the vitamin $\mathrm{C}$ and $\mathrm{E}$ studies and analyzed in the present review can also be seen with other antioxidants. For example, supplementation with $\mathrm{N}$-acetylcysteine or ubiquinone-10 reported to attenuate the adaptive responses to exercise $[22,52]$, spirulina or quercetin to enhance the adaptive responses to exercise $[53,54]$ as well as green tea extract or coffee berries to exert no effect on adaptations to exercise $[55,56]$. There is a tendency to include molecules with heterogeneous properties, such as those presented previously, under the general heading of "antioxidants." This approach might lead to erroneous interpretations, because these molecules have different mechanisms of action and the antioxidant activity is only one of their functions, if any [57]. Therefore, one should avoid extrapolating the results from one antioxidant to another.

Why is there so much divergence among studies addressing the effect of vitamin $\mathrm{C}$ and/or $\mathrm{E}$ supplementation on exercise adaptations? We believe that the main reason is the near uniqueness of each study in terms of type of exercise (aerobic or anaerobic), species (rat or humans), age (young or old), tissue (blood or muscle), oxidant biomarker, and training endpoints examined. Other factors that could explain some of the diversity of results include nutrition, subject characteristics, exercise characteristics, and experimental error arising from the complexity of the techniques employed in redox status analysis. Finally, the lack of consensus may be partially explained by the biological variability of redox biochemistry. Thus, it is desirable to develop and achieve standardization and agreement on key influencing factors which investigators should employ when designing studies in this area of biology.

Except for the methodological factors that may be responsible for the lack of a consensus among the studies, an appropriate conceptual framework may also be needed to integrate the disparate exercise adaptations to antioxidant supplementation. Hormesis is a dose-response phenomenon characterized by either a U-shaped or an inverted Ushaped dose response depending on the endpoint measured $[58,59]$. In hormesis, dose response is characterized by low-dose stimulation and high-dose inhibition, leading to the biphasic, hormetic dose-response curve [58, 59]. The conceptual framework provided by hormesis can potentially reconcile differences that emerge among relevant studies regarding the effect of antioxidant supplementation on exercise adaptations. The hormetic concept predicts that the effects of antioxidant supplementation on muscle performance and redox homeostasis are dependent on the antioxidant dose. In fact, the limited evidence indicates that too much generation of reactive species may be harmful while the modest generation may be beneficial [60]. Based on this evidence, it is plausible to assume that the divergence regarding the effects of antioxidant supplementation on exercise adaptations and redox homeostasis may partly be explained by the different degrees of reactive species decomposition. Despite the promising first data and the appealing nature of the hormesis concept, the establishment of deviation from linearity in dose-response relationships for exercise-induced alterations in redox homeostasis requires studies specifically designed to locate and describe the possible hormetic effects of exercise.

Collectively, it becomes challenging to draw clear conclusions surrounding the impact of vitamins $\mathrm{C}$ and $\mathrm{E}$ on adaptations to chronic exercise. While the selected hypotheses from these studies may seem clear and ripe for comparisons, too many differences exist among the employed methodological approaches to derive definitive conclusions regarding the influence of vitamin $\mathrm{C}$ and $\mathrm{E}$ supplementation on adaptation to chronic exercise. Therefore, we suggest that investigating colleagues of this work direct stronger attention on the following key factors:

(1) selection and detailed description of the appropriate training stimulus,

(2) determination of valid and reliable redox biomarkers such as isoprostanes and glutathione using appropriate techniques and assays,

(3) measurement of biomarkers in the same body fluids and cell materials,

(4) collection of specimens at multiple time points after exercise,

(5) application of antioxidant supplementation on already well-trained individuals,

(6) assessment of performance through valid and reliable testing procedures,

(7) assessment of training adaptations through the use of valid and reliable biochemical and physiological markers,

(8) administration of a specific dosage of vitamins $C$ and $\mathrm{E}$ at a specified time during the training period,

(9) assessment of dietary intake of redox-active nutrients like antioxidants,

(10) evaluation and reporting of hydration status. 


\section{Conclusion and Future Perspectives}

Recent studies that employed both animal and human models provide conflicting and confusing outcomes regarding the efficacy of vitamin $C$ and $E$ supplementation on training adaptations. A close examination of this literature reveals many situations in which methodological differences are likely contributors to the widely varied outcomes. In consideration of these shortcomings, this paper was developed to highlight key differences in the literature and state the need to develop a more widespread systematic approach to investigations in this area. Recommendations regarding key methodological considerations are provided, and it is our hope that future researchers can use this paper as a reference or an area of basis upon which to build future studies which result in consistent and meaningful outcomes that will facilitate and optimize the findings in this important area of exercise biology. Based on the conflicting evidence regarding the effects of higher intakes of vitamin $\mathrm{C}$ and/or $\mathrm{E}$ on exercise performance and redox homeostasis, a permanent intake of nonphysiological dosages of vitamin $\mathrm{C}$ and/or E should not be recommended to healthy individuals. This must not be confused with a high intake of fruit and vegetables, which is considered safe and beneficial.

\section{References}

[1] M. P. Bradshaw, C. Barril, A. C. Clark, P. D. Prenzler, and G. R. Scollary, "Ascorbic acid: a review of its chemistry and reactivity in relation to a wine environment," Critical Reviews in Food Science and Nutrition, vol. 51, no. 6, pp. 479-498, 2011.

[2] Z. Jiang, X. Yin, and Q. Jiang, "Natural forms of vitamin E and 13'-carboxychromanol, a long-chain vitamin E metabolite, inhibit leukotriene generation from stimulated neutrophils by blocking calcium influx and suppressing 5-lipoxygenase activity, respectively," Journal of Immunology, vol. 186, no. 2, pp. 1173-1179, 2011.

[3] M. J. Jackson, D. Pye, and J. Palomero, "The production of reactive oxygen and nitrogen species by skeletal muscle," Journal of Applied Physiology, vol. 102, no. 4, pp. 1664-1670, 2007.

[4] S. K. Powers, J. Duarte, A. N. Kavazis, and E. E. Talbert, "Reactive oxygen species are signalling molecules for skeletal muscle adaptation," Experimental Physiology, vol. 95, no. 1, pp. $1-9,2010$.

[5] R. J. Bloomer and W. A. Smith, "Oxidative stress in response to aerobic and anaerobic power testing: influence of exercise training and carnitine supplementation," Research in Sports Medicine, vol. 17, no. 1, pp. 1-16, 2009.

[6] T. T. Peternelj and J. S. Coombes, "Antioxidant supplementation during exercise training: beneficial or detrimental?" Sports Medicine, vol. 41, no. 12, pp. 1043-1069, 2011.

[7] S. K. Powers, L. L. Ji, and C. Leeuwenburgh, "Exercise traininginduced alterations in skeletal muscle antioxidant capacity: a brief review," Medicine and Science in Sports and Exercise, vol. 31, no. 7, pp. 987-997, 1999.

[8] J. Sobal and L. F. Marquart, "Vitamin/mineral supplement use among athletes: a review of the literature," International Journal of Sport Nutrition, vol. 4, no. 4, pp. 320-334, 1994.

[9] M. C. Gomez-Cabrera, E. Domenech, M. Romagnoli et al., "Oral administration of vitamin C decreases muscle mitochondrial biogenesis and hampers training-induced adaptations in endurance performance," American Journal of Clinical Nutrition, vol. 87, no. 1, pp. 142-149, 2008.

[10] M. Ristow, K. Zarse, A. Oberbach et al., "Antioxidants prevent health-promoting effects of physical exercise in humans," Proceedings of the National Academy of Sciences of the United States of America, vol. 106, no. 21, pp. 8665-8670, 2009.

[11] K. Higashida, S. H. Kim, M. Higuchi, J. O. Holloszy, and D. H. Han, "Normal adaptations to exercise despite protection against oxidative stress," American Journal of Physiology Endocrinology and Metabolism, vol. 301, no. 5, pp. E779-E784, 2011.

[12] L. A. Roberts, K. Beattie, G. L. Close, and J. P. Morton, "Vitamin C consumption does not impair training-induced improvements in exercise performance," International Journal of Sports Physiology and Performance, vol. 6, no. 1, pp. 58-69, 2011.

[13] M. J. Ryan, H. J. Dudash, M. Docherty et al., "Vitamin E and $\mathrm{C}$ supplementation reduces oxidative stress, improves antioxidant enzymes and positive muscle work in chronically loaded muscles of aged rats," Experimental Gerontology, vol. 45, no. 11, pp. 882-895, 2010.

[14] A. A. Theodorou, M. G. Nikolaidis, V. Paschalis et al., "No effect of antioxidant supplementation on muscle performance and blood redox status adaptations to eccentric training," American Journal of Clinical Nutrition, vol. 93, no. 6, pp. 13731383, 2011.

[15] C. Yfanti, T. Åkerström, S. Nielsen et al., "Antioxidant supplementation does not alter endurance training adaptation," Medicine and Science in Sports and Exercise, vol. 42, no. 7, pp. 1388-1395, 2010.

[16] C. Yfanti, C. P. Fischer, S. Nielsen, T. Akerstrom, A. R. Nielsen, A. S. Veskoukis et al., "Role of vitamin C and E supplementation on IL-6 in response to training," Journal of Applied Physiology, vol. 112, no. 6, pp. 990-1000, 2012.

[17] C. Yfanti, A. R. Nielsen, T. Åkerström et al., "Effect of antioxidant supplementation on insulin sensitivity in response to endurance exercise training," American Journal of Physiology, vol. 300, no. 5, pp. E761-E770, 2011.

[18] D. M. Bailey, C. Williams, J. A. Betts, D. Thompson, and T. L. Hurst, "Oxidative stress, inflammation and recovery of muscle function after damaging exercise: effect of 6-week mixed antioxidant supplementation," European Journal of Applied Physiology, vol. 111, no. 6, pp. 925-936, 2011.

[19] J. S. Coombes, S. K. Powers, B. Rowell et al., "Effects of vitamin E and $\alpha$-lipoic acid on skeletal muscle contractile properties," Journal of Applied Physiology, vol. 90, no. 4, pp. 1424-1430, 2001.

[20] C. L. Dumke, D. C. Nieman, A. C. Utter et al., "Quercetin's effect on cycling efficiency and substrate utilization," Applied Physiology, Nutrition and Metabolism, vol. 34, no. 6, pp. 9931000, 2009.

[21] M. C. Gomez-Cabrera, A. Martínez, G. Santangelo, F. V. Pallardó, J. Sastre, and J. Viña, "Oxidative stress in marathon runners: interest of antioxidant supplementation," The British Journal of Nutrition, vol. 96, supplement 1, pp. S31-33, 2006.

[22] C. Malm, M. Svensson, B. Ekblom, and B. Sjödin, "Effects of ubiquinone-10 supplementation and high intensity training on physical performance in humans," Acta Physiologica Scandinavica, vol. 161, no. 3, pp. 379-384, 1997.

[23] C. Malm, M. Svensson, B. Sjöberg, B. Ekblom, and B. Sjödin, "Supplementation with ubiquinone-10 causes cellular damage during intense exercise," Acta Physiologica Scandinavica, vol. 157, no. 4, pp. 511-512, 1996. 
[24] D. C. Nieman, D. A. Henson, K. R. Maxwell et al., "Effects of quercetin and EGCG on mitochondrial biogenesis and immunity," Medicine and Science in Sports and Exercise, vol. 41, no. 7, pp. 1467-1475, 2009.

[25] D. W. Wray, A. Uberoi, L. Lawrenson, D. M. Bailey, and R. S. Richardson, "Oral antioxidants and cardiovascular health in the exercise-trained and untrained elderly: a radically different outcome," Clinical Science, vol. 116, no. 5, pp. 433-441, 2009.

[26] D. Njus and P. M. Kelley, "Vitamins C and E donate single hydrogen atoms in vivo," FEBS Letters, vol. 284, no. 2, pp. $147-$ 151, 1991.

[27] G. R. Buettner, "The pecking order of free radicals and antioxidants: lipid peroxidation, $\alpha$-tocopherol, and ascorbate," Archives of Biochemistry and Biophysics, vol. 300, no. 2, pp. 535-543, 1993.

[28] B. Halliwell and J. Gutteridge, Free Radicals in Biology and Medicine, Oxford University Press, New York, NY, USA, 2007.

[29] S. Asha Devi, S. Prathima, and M. V. Subramanyam, "Dietary vitamin E and physical exercise: I. Altered endurance capacity and plasma lipid profile in ageing rats," Experimental Gerontology, vol. 38, no. 3, pp. 285-290, 2003.

[30] S. Asha Devi, S. Prathima, and M. V. Subramanyam, "Dietary vitamin E and physical exercise: II. Antioxidant status and lipofuscin-like substances in aging rat heart," Experimental Gerontology, vol. 38, no. 3, pp. 291-297, 2003.

[31] J. O. Holloszy, K. Higashida, S. H. Kim, M. Higuchi, and D. H. Han, "Response to letter to the editor by GomezCabrera et al.", American Journal of Physiology Endocrinology and Metabolism, vol. 302, pp. E478-E479, 2012.

[32] M. G. Nikolaidis, A. Z. Jamurtas, V. Paschalis, I. G. Fatouros, Y. Koutedakis, and D. Kouretas, "The effect of muscledamaging exercise on blood and skeletal muscle oxidative stress: magnitude and time-course considerations," Sports Medicine, vol. 38, no. 7, pp. 579-606, 2008.

[33] M. G. Nikolaidis, A. Kyparos, C. Spanou, V. Paschalis, A. A. Theodorou, and I. S. Vrabas, "Redox biology of exercise: an integrative and comparative consideration of some overlooked issues," The Journal of Experimental Biology, vol. 215, no. 10, pp. 1615-1625, 2012.

[34] M. G. Nikolaidis, V. Paschalis, G. Giakas et al., "Decreased blood oxidative stress after repeated muscle-damaging exercise," Medicine and Science in Sports and Exercise, vol. 39, no. 7, pp. 1080-1089, 2007.

[35] G. R. Buettner, "The pecking order of free radicals and antioxidants: lipid peroxidation, $\alpha$-tocopherol, and ascorbate," Archives of Biochemistry and Biophysics, vol. 300, no. 2, pp. 535-543, 1993.

[36] A. A. Gaeini, N. Rahnama, and M. R. Hamedinia, "Effects of vitamin E supplementation on oxidative stress at rest and after exercise to exhaustion in athletic students," Journal of Sports Medicine and Physical Fitness, vol. 46, no. 3, pp. 458-461, 2006.

[37] D. C. Nieman, D. A. Henson, S. R. McAnulty et al., "Influence of vitamin $\mathrm{C}$ supplementation on oxidative and immune changes after an ultramarathon," Journal of Applied Physiology, vol. 92, no. 5, pp. 1970-1977, 2002.

[38] V. H. Teixeira, H. F. Valente, S. I. Casal, A. F. Marques, and P. A. Moreira, "Antioxidants do not prevent postexercise peroxidation and may delay muscle recovery," Medicine and Science in Sports and Exercise, vol. 41, no. 9, pp. 1752-1760, 2009.

[39] D. Thompson, C. Williams, P. Garcia-Roves, S. J. McGregor, F. McArdle, and M. J. Jackson, "Post-exercise vitamin C supplementation and recovery from demanding exercise," European Journal of Applied Physiology, vol. 89, no. 3-4, pp. 393-400, 2003.

[40] D. Thompson, C. Williams, M. Kingsley et al., "Muscle soreness and damage parameters after prolonged intermittent shuttle-running following acute vitamin C supplementation," International Journal of Sports Medicine, vol. 22, no. 1, pp. 6875, 2001.

[41] N. G. Avery, J. L. Kaiser, M. J. Sharman, T. P. Scheett, D. M. Barnes, A. L. Gomez et al., "Effects of vitamin E supplementation on recovery from repeated bouts of resistance exercise," Journal of Strength and Conditioning Research, vol. 17, no. 4, pp. 801-809, 2003.

[42] S. C. Bryer and A. H. Goldfarb, "Effect of high dose vitamin C supplementation on muscle soreness, damage, function, and oxidative stress to eccentric exercise," International Journal of Sport Nutrition and Exercise Metabolism, vol. 16, no. 3, pp. 270-280, 2006.

[43] S. R. J. Maxwell, P. Jakeman, H. Thomason, C. Leguen, and G. H. G. Thorpe, "Changes in plasma antioxidant status during eccentric exercise and the effect of vitamin supplementation," Free Radical Research Communications, vol. 19, no. 3, pp. 191202, 1993.

[44] J. M. McBride, W. J. Kraemer, T. Triplett-McBride, and W. Sebastianelli, "Effect of resistance exercise on free radical production," Medicine and Science in Sports and Exercise, vol. 30, no. 1, pp. 67-72, 1998.

[45] J. M. Sacheck, P. E. Milbury, J. G. Cannon, R. Roubenoff, and J. B. Blumberg, "Effect of vitamin E and eccentric exercise on selected biomarkers of oxidative stress in young and elderly men," Free Radical Biology and Medicine, vol. 34, no. 12, pp. 1575-1588, 2003.

[46] M. Lamprecht, P. Hofmann, J. F. Greilberger, and G. Schwaberger, "Increased lipid peroxidation in trained men after 2 weeks of antioxidant supplementation," International Journal of Sport Nutrition and Exercise Metabolism, vol. 19, no. 4, pp. 385-399, 2009.

[47] D. C. Nieman, D. A. Henson, S. R. McAnulty et al., "Vitamin E and immunity after the Kona Triathlon World Championship," Medicine and Science in Sports and Exercise, vol. 36, no. 8, pp. 1328-1335, 2004.

[48] R. J. Bloomer, M. J. Falvo, A. C. Fry, B. K. Schilling, W. A. Smith, and C. A. Moore, "Oxidative stress response in trained men following repeated squats or sprints," Medicine and Science in Sports and Exercise, vol. 38, no. 8, pp. 14361442, 2006.

[49] T. M. Farney, C. G. McCarthy, R. E. Canale, B. K. Schilling, P. N. Whitehead, and R. J. Bloomer, "Absence of blood oxidative stress in trained men following strenuous exercise," Medicine and Science in Sports and Exercise. In press.

[50] Y. A. Shin, J. H. Lee, W. Song, and T. W. Jun, "Exercise training improves the antioxidant enzyme activity with no changes of telomere length," Mechanisms of Ageing and Development, vol. 129, no. 5, pp. 254-260, 2008.

[51] S. Ayres, J. Baer, and M. T. R. Subbiah, "Exercised-induced increase in lipid peroxidation parameters in amenorrheic female athletes," Fertility and Sterility, vol. 69, no. 1, pp. 7377, 1998.

[52] A. C. Petersen, M. J. McKenna, I. Medved, K. T. Murphy, M. J. Brown, P. Della Gatta et al., "Infusion with the antioxidant Nacetylcysteine attenuates early adaptive responses to exercise in human skeletal muscle," Acta Physiologica, vol. 204, no. 3, pp. 382-392, 2012. 
[53] M. Kalafati, A. Z. Jamurtas, M. G. Nikolaidis et al., "Ergogenic and antioxidant effects of spirulina supplementation in humans," Medicine and Science in Sports and Exercise, vol. 42, no. 1, pp. 142-151, 2010.

[54] D. C. Nieman, A. S. Williams, R. A. Shanely et al., "Quercetin's influence on exercise performance and muscle mitochondrial biogenesis," Medicine and Science in Sports and Exercise, vol. 42, no. 2, pp. 338-345, 2010.

[55] P. Eichenberger, S. Mettler, M. Arnold, and P. C. Colombani, "No effects of three-week consumption of a green tea extract on time trial performance in endurance-trained men," International Journal for Vitamin and Nutrition Research, vol. 80, no. 1, pp. 54-64, 2010.

[56] S. M. Ostojic, M. D. Stojanovic, B. Djordjevic, M. Jourkesh, and N. Vasiljevic, "The effects of a 4-week coffeeberry supplementation on antioxidant status, endurance, and anaerobic performance in college athletes," Research in Sports Medicine, vol. 16, no. 4, pp. 281-294, 2008.

[57] C. Fortes and F. Virgili, "Antioxidant vitamins are not "just antioxidants": not necessarily harmful when targeted to the right population," BioFactors, vol. 33, no. 3, pp. 177-180, 2008.

[58] E. J. Calabrese and L. A. Baldwin, "Hormesis: U-shaped dose responses and their centrality in toxicology," Trends in Pharmacological Sciences, vol. 22, no. 6, pp. 285-291, 2001.

[59] Z. Radak, H. Y. Chung, and S. Goto, "Exercise and hormesis: oxidative stress-related adaptation for successful aging," Biogerontology, vol. 6, no. 1, pp. 71-75, 2005.

[60] Z. Radak, H. Y. Chung, E. Koltai, A. W. Taylor, and S. Goto, "Exercise, oxidative stress and hormesis," Ageing Research Reviews, vol. 7, no. 1, pp. 34-42, 2008. 


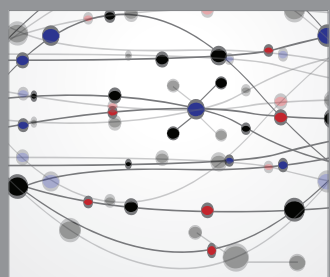

The Scientific World Journal
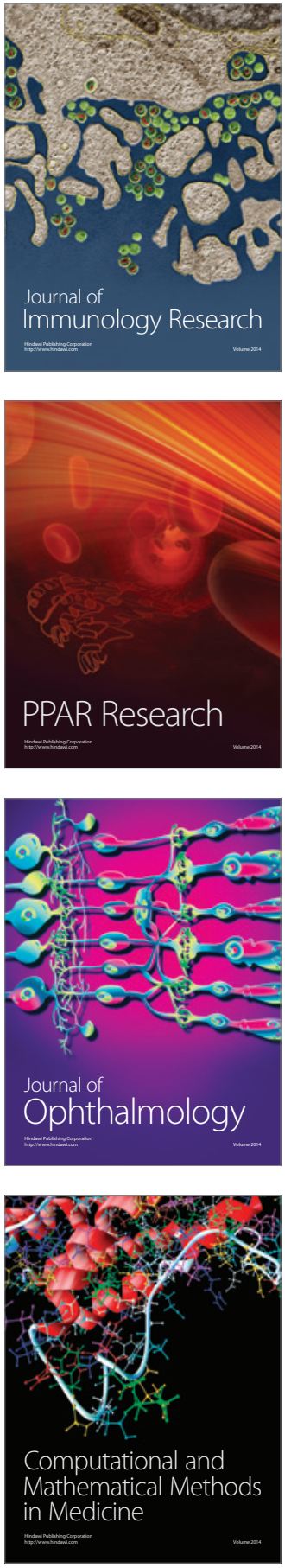

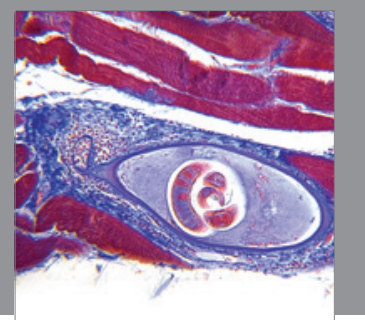

Gastroenterology

Research and Practice
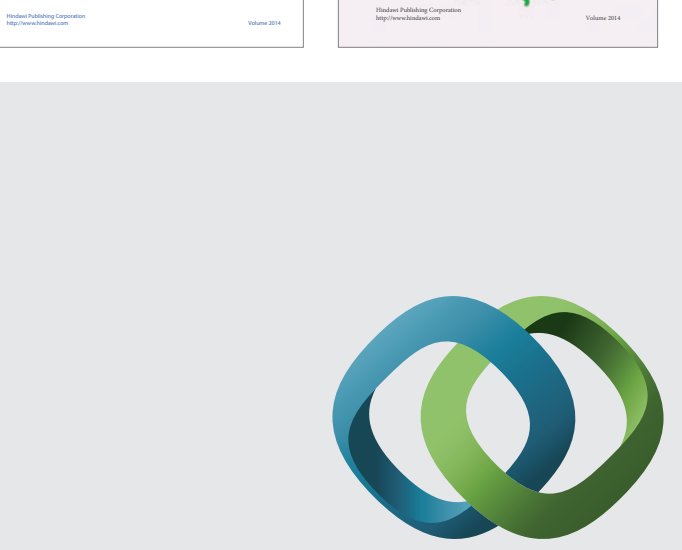

\section{Hindawi}

Submit your manuscripts at

http://www.hindawi.com
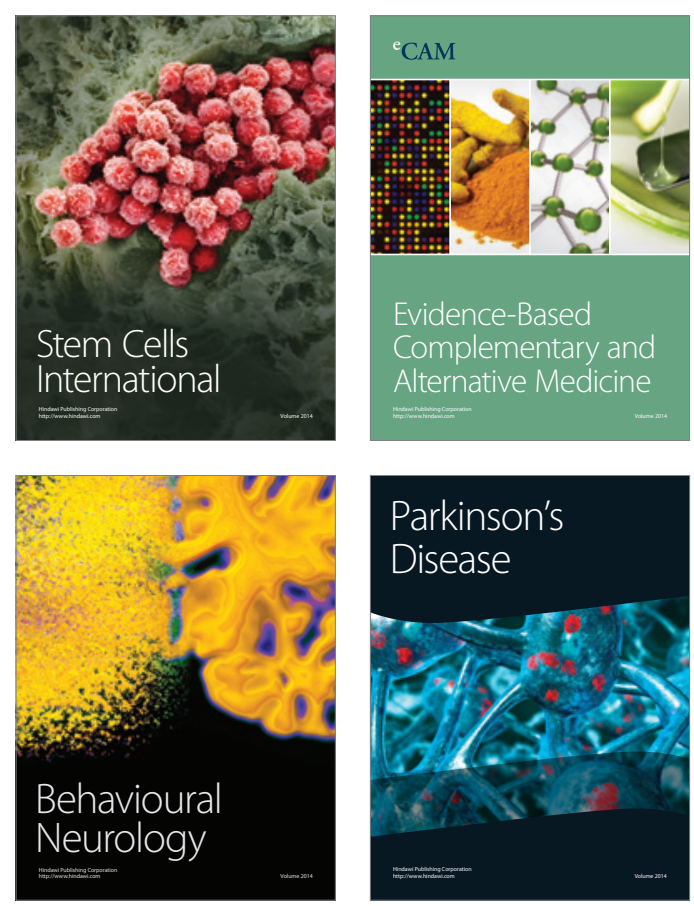

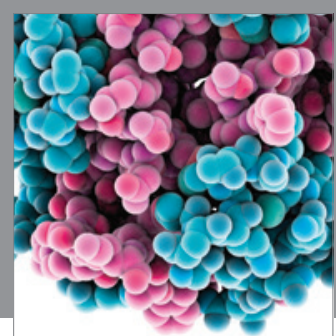

Journal of
Diabetes Research

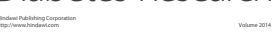

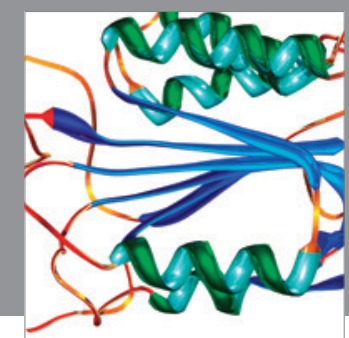

Disease Markers
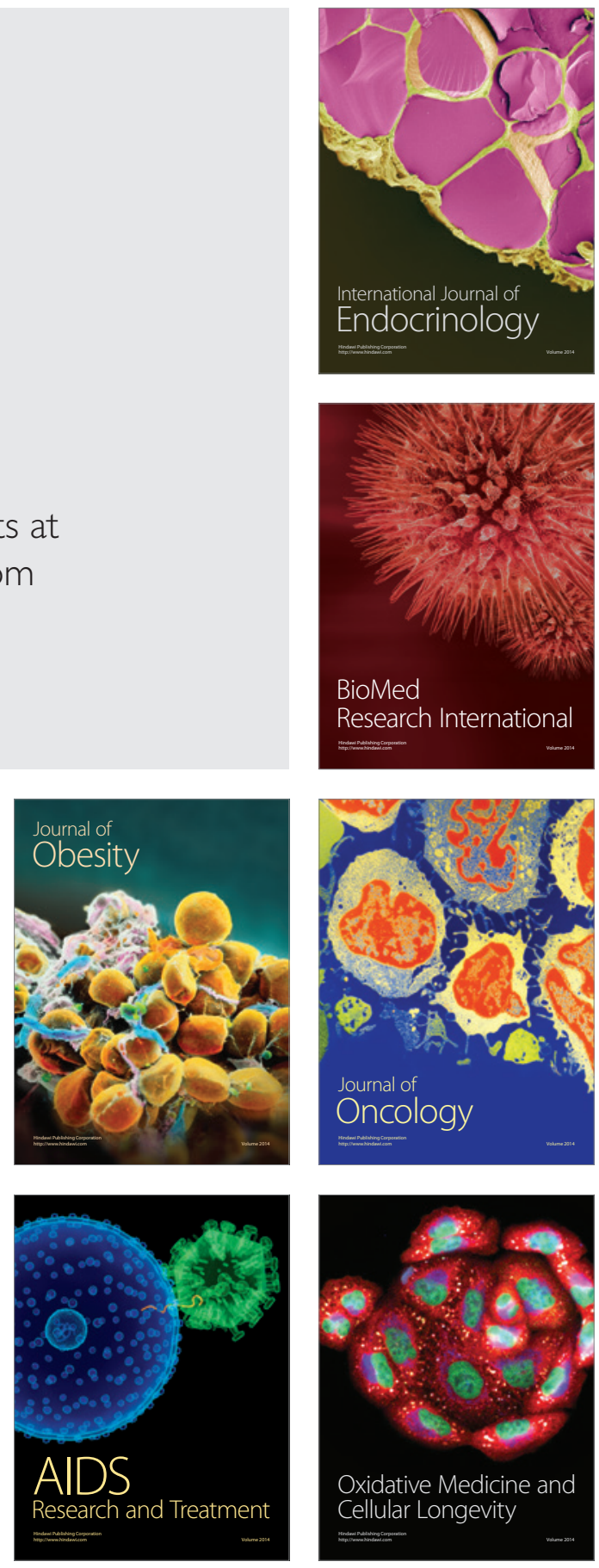